\title{
Whole-Body [18F]-FDG-PET/MRI for Oncology: A Consensus Recommendation*
}

\section{Konsensempfehlungen zur Anwendung der Ganzkörper [18F]-FDG-PET/MRT in der onkologischen Bildgebung}

Authors

Lale Umutlu', Thomas Beyer², Johannes Stefan Grueneisen¹, Christoph Rischpler ${ }^{3}$, Harald H. Quick ${ }^{4}$, Patrick Veit-Haibach ${ }^{5}$, Matthias Eiber ${ }^{6}$, Sandra Purz ${ }^{7}$, Gerald Antoch ${ }^{8}$, Sergios Gatidis ${ }^{9}$, Konstantin Nikolaou ${ }^{9}$, Jürgen F. Schaefer ${ }^{9}$, Ivo Rausch², Ken Herrmann ${ }^{3}$

Vorstand der Interdisziplinären AG für Hybride Bildgebung in alphabetischer Reihenfolge:

K. Herrmann ${ }^{3}$, B. J. Krause ${ }^{10}$, S. O. Schoenberg ${ }^{11}$, L. Umutlu ${ }^{1}$

Vorstand der Deutschen Röntgengesellschaft (DRG) und der Deutschen Gesellschaft für Nuklearmedizin (DGN) in alphabetischer Reihenfolge:

F. Anton ${ }^{12}$, G Antoch ${ }^{8}$, M. Hacker ${ }^{13}$, B. J. Krause ${ }^{10}$, M. Luster $^{14}$, S. Neumann ${ }^{12}$, S. O. Schoenberg ${ }^{11}$, D. Vorwerk ${ }^{15}$

Affiliations

1 Department of Diagnostic and Interventional Radiology and Neuroradiology, University-Hospital Essen, Germany

2 QIMP Group, Centre for Medical Imaging and Biomedical Engineering, Medical University of Vienna, Austria

3 Department of Nuclear Medicine, University-Hospital Essen, Germany

4 High-Field- and Hybrid-MR-Imaging, University-Hospital Essen, Germany

5 Joint Department of Medical Imaging, University of Toronto, Canada

6 Department of Nuclear Medicine, Klinikum rechts der Isar, Technical University of Munich, Germany

7 Department of Nuclear Medicine, University of Leipzig, Germany

8 Department of Diagnostic and Interventional Radiology, University Düsseldorf, Medical Faculty, Düsseldorf, Germany

9 Department of Diagnostic and Interventional Radiology, University-Hospital Tübingen, Germany

10 Department of Nuclear Medicine, University Medical Center Rostock, Germany

11 Department of Clinical Radiology and Nuclear Medicine, University Medical Center Mannheim, Germany

12 Deutsche Röntgengesellschaft, Berlin, Germany

13 Division of Nuclear Medicine, Department of Biomedical Imaging and Image-guided Therapy, Medical University Vienna, Austria

14 Department of Nuclear Medicine, University Hospital Marburg, Germany

15 Department of Radiology, Klinikum Ingolstadt, Germany
Für die DRG

Prof. Dr. Stefan Schönberg, Präsident

Prof. Dr. Dierk Vorwerk, stellvertrender Präsident

Prof. Dr. Gerald Antoch, President-elect

Dr. Frank Anton, Schatzmeister

Dr. Stefan Neumann, Schriftführer

Für die DGN

Prof. Dr Bernd-Joachim Krause, Präsident

Prof. Dr. Markus Luster, Marburg, stellvertretender Präsident Prof. Dr. Marcus Hacker, AKH Wien, Schriftführer

Key words

$\mathrm{PET} / \mathrm{MRI}$, oncology, consensus recommendations, whole-body imaging, hybrid imaging

received 08.10.2018

accepted 22.12.2018

Bibliography

DOI https://doi.org/10.1055/a-0828-8654

Published online: 28.2.2019

Fortschr Röntgenstr 2019; 191: 289-297

(c) Georg Thieme Verlag KG, Stuttgart · New York

ISSN 1438-9029

Correspondence

Prof. Dr. Lale Umutlu

Institut für Diagnostische und Interventionelle Radiologie und Neuroradiologie, Universitätsklinik Essen, Hufelandstr. 55.

45147 Essen, Germany

Tel.: ++ 49/2 01/7 231501

Fax: $++49 / 201 / 7231548$

lale.umutlu@uk-essen.de

* Published simultaneously in Nuklearmedizin 2019; DOI: 10.1055/ a-0830-4453. 


\section{ZUSAMMENFASSUNG}

Nach initialen Plänen zur Einführung integrierter PET/MRTSysteme im Jahre 2006 standen 2010 die ersten kommerziell erwerbbaren Scanner für die klinische Anwendung am Patienten zur Verfügung. Sie folgten damit früheren, bereits etablierten hybriden Bildgebungsmodalitäten, wie der PET/CT und SPECT/CT, bei denen der Vorteil einer kombinierten Analyse molekularer und anatomischer Parameter zur Beantwortung onkologischer Fragestellungen gezeigt werden konnte. Bis dato wurden weltweit ca. 150 PET/MRT-Systeme in Kliniken, Praxen und diversen Forschungsinstitutionen installiert. Anhand der aktuellen Studienlage lässt sich die onkologische Diagnostik weiterhin als Hauptanwendungsgebiet erkennen. Aufgrund der zunehmenden Anwendung der PET/MRT in der Patientenversorgung besteht nun die Notwendigkeit einer Anpassung der Arbeitsabläufe an die Anforderungen des klinischen Alltags sowie einer Standardisierung der Untersuchungsprotokolle an die spezifischen medizinischen Fragestellungen. In dem vorliegenden Manuskript werden Konsensempfehlungen für die Indikationsstellung und Vorbereitung des Patienten sowie die Durchführung und Interpretation einer PET/MRT-Untersuchung dargestellt. Darüber hinaus werden Untersuchungsprotokolle zur Anwendung der Ganzkörper [18F]-FDG-PET/MRT zusammengefasst. Diese Empfehlungen wurden durch Experten in den Bereichen der PET, MRT und PET/MRT-Bildgebung zusammengestellt. Sie sollen zur Standardisierung der [18F]-FDG-PET/MRT-Diagnostik onkologischer Patienten und zu einer breiteren klinischen Akzeptanz dieser Bildgebungsmodalität zum Wohle der Patienten beitragen.
ABSTRACT

Combined PET/MR imaging (PET/MRI) was proposed for patient management in 2006 with first commercial versions of integrated whole-body systems becoming available as of 2010. PET/MRI followed the prior evolution of hybrid imaging as attested by the successful adoption of combined PET/CT and SPECT/CT since the early $2000 \mathrm{~s}$. Today, around 150 whole-body PET/MRI systems have become operational worldwide. One of the main application fields of PET/MRI is oncologic imaging. Despite the increasing use of PET/MRI, little governance regarding standardized PET/MRI protocols has been provided to date. Standardization and harmonization of imaging protocols is, however, mandatory for efficient on-site patient management and multi-center studies. This document summarizes consensus recommendations on key aspects of patient referral and preparation, PET/MRI workflow and imaging protocols, as well as reporting strategies for whole-body [18F]-FDG-PET/MRI. These recommendations were created by early adopters and key experts in the field of PET, MRI and PET/MRI. This document is intended to provide guidance for the harmonization and standardization of PET/ MRI today and to support wider clinical adoption of this imaging modality for the benefit of patients.

\section{Citation Format}

- Umutlu L, Beyer T, Grueneisen JS et al. Whole-Body [18F]FDG-PET/MRI for Oncology: A Consensus Recommendation. Fortschr Röntgenstr 2019; 191: 289-297

\section{Introduction}

The aim of this consensus recommendation is to provide guidance to healthcare experts and physicians regarding clinical indications, execution and interpretation of [18F]-Fluorodeoxyglucose (FDG) Positron emission tomography/magnetic resonance imaging examinations ([18F]-FDG PET/MRI) for whole-body staging in oncology [1].

$\mathrm{PET}$ is a noninvasive imaging technique that provides quantitative information on 3-dimensional distributions of radioactively labelled biomolecules (tracer) in tissues. [18F]-FDG is a tracer composed of radiolabeled glucose, which is the most common tracer for oncology imaging indications [2]. For the majority of tumors, malignant cells display activated glycolytic pathways resulting in increased glucose utilization via upregulation of glucose transporter expression and hexokinase activity [3, 4]. Thus, more of the glucose analog, [18F]-FDG, is taken up in metabolically active cancerous cells than in surrounding healthy tissues. Therefore, [18F]-FDG-PET has been demonstrated to be a sensitive method and well-established imaging modality for detection, re-/staging as well as for the evaluation of therapy response of solid tumors $[5,6]$.
Magnetic resonance imaging (MRI) is a noninvasive imaging technique that provides anatomical $3 \mathrm{D}$ visualization of tissues with high spatial resolution based on relative differences in resonance frequencies of spins following external excitation [7]. In addition, MRI employs multiple imaging sequences and associated soft-tissue contrasts that yield noninvasive insight into functional and cellular aspects of tissues and organs [8]. The magnetic fieldbased excitation and resonance measurement method sets MRI apart from computed tomography (CT), which is a pure transmission method based on the attenuation of ionizing radiation. In contrast to CT-based transmission imaging, MRI does not employ ionizing radiation. Thus, the exposure of patients undergoing PET/ MRI to ionizing radiation originates from the PET portion only and therefore is significantly lower compared to PET/CT [9].

While attenuation correction is a well-established aspect of PET/CT imaging, it was a methodologically challenging task to overcome in integrated PET/MRI (please also refer to the section "Attenuation correction"). Thus, the introduction of MR-compatible PET detector systems provided the basis for the hardware integration of PET and MRI components into a single, integrated system $[10,11]$. Prior work of developers of small-animal imaging systems [12] has helped to replace the photomultipliers in PET detectors with semiconductor-based diodes that are capable of am- 
plifying the scintillation signal in the scintillator crystals without being affected by the magnetic field [13]. Following further technical and methodological work, fully integrated PET/MRI systems have been introduced for clinical use with a magnetic field strength of $3 \mathrm{~T}$ [14] and MRI sequences were developed that enable a reliable correction of attenuation artifacts in PET with comparable quality to $\mathrm{CT}$ transmission maps for PET/CT imaging (please also refer to section "Attenuation correction").

At present, the number of clinical studies with PET/MRI is continuously increasing. Recent publications comparing the diagnostic accuracy of whole-body PET/MRI demonstrated equivalence to that of PET/CT (using the same tracers) $[15,16]$. However, a number of potential benefits for PET/MRI have been highlighted with regards to the high soft-tissue contrast of MRI and consecutively improved delineation of tumorous lesions [17-20]. Such studies will benefit from overcoming of the existing variations in the use of PET/MRI for distinct diagnostic questions [21], and, therefore, consensus recommendations are mandatory to limit these a priori variations through harmonization and standardization approaches.

\section{Definitions}

Similar to the endeavors to establish consensus recommendations for combined PET/CT imaging protocols and definitions [6, 22, 23], we will use the following definitions for combined PET/MRI for easier understanding:

- A combined PET/MRI system is an integrated PET and MRI system that enables the generation of PET and MRI data during the same patient acquisition without the need to reposition the patient between examinations.

- Fully integrated PET/MRI refers to a hardware combination of both imaging systems that permits the simultaneous acquisition of PET and MRI data, requiring the use of MRI-compatible PET detectors.

- PET/MRI and MR/PET can be used interchangeably. The same is true for PET-MRI and MRI-PET.

- The information contained in the images from a PET/MRI examination is given by the tracer-of-choice, the method of acquisition of the emission data (static or dynamic mode) and the mode of the MR acquisition (T1-weighting, T2-weighting, dynamic contrast-enhanced sequences, proton density, diffusion-weighted imaging, arterial spin labelling, apparent diffusion coefficient, etc.).

- In the clinical routine, PET/MRI examinations do not include a transmission measurement, and, therefore, alternative means have to be provided to derive attenuation correction factors (ACF) for the PET data in order to quantify the molecular signals [24].

- Artifacts comprise all types of PET and MR image distortions that include visually perceived deviations from typical representations of anatomy and function that may or may not cause a quantitative bias (e. g., lesion size, tracer concentration, etc.). These distortions are likely not to arise from a disease process but from methodological pitfalls or system malfunctions [25].

\section{Indications for/application fields of PET/MRI}

The following indications/application fields apply to whole-body [18F]-FDG-PET/MRI examinations in oncologic imaging.

- Staging/restaging of known tumors

- Detection/exclusion of tumor relapse

- Therapy monitoring

- Detection of a cancer of unknown primary (CUP)

- Further differentiation of indeterminate findings in conventional imaging

- Radiation therapy or biopsy planning

\section{PET/MRI examination}

\section{Necessary patient information}

- In preparation for [18F]-FDG-PET/MRI examinations, the following information should be collected from the patient:

- History focused on the type and location of the malignant disease,

- Date of the initial diagnosis,

- Type of diagnostic confirmation,

- Treatment prior to the current PET/MRI scan (e. g. biopsy date and results, histology, surgery, radiotherapy, chemotherapy),

- Medication at the time of examination, and

- Any prior examination (particularly imaging studies)

- History of diabetes mellitus, last food intake, infections or recent colds

- Ability of the patient to lie still for the duration of the scan (30-60 min)

- Claustrophobia: Ability of the patient to remain in the PET/MRI system for the duration of the examination

- Ability to provide informed consent

\section{Patient preparation}

The main objectives of patient preparation are the reduction of tracer uptake in normal tissue (e.g. heart, skeletal muscle) while preserving tracer uptake in the target structures (tumor tissue). The following is a general-use protocol:

- Prior to the examination:

- Patients are advised not to eat or drink any food (excluding water) four to six hours prior to the application of [18F]FDG in order to reduce physiologic blood sugar levels and ensure low serum insulin levels. Sufficient hydration is recommended. Parenteral nutrition or glucose-containing infusions also have to be discontinued four to six hours prior to the application of the radiotracer.

- If the use of MR contrast agents is planned, the respective contraindications and applicable restrictions (e. g., potential allergic reactions to Gadolinium-based contrast agents, kidney disease or renal dysfunction) must be taken into consideration. Caution needs to be taken in the case of elevated creatinine levels or reduced glomerular filtration rates, indicating renal insufficiency [26, 27]. 
- Patients have to discard all removable metal objects (e. g., rings, piercings, medication pumps, etc.) before entering the PET/MRI examination room. In case of implanted ferromagnetic devices (e. g., pacemakers, ICD, LVAD, event recorders, stents, metal plates from orthopedic interventions, etc.) or metal splinters/shrapnel, PET/MRI examinations should only be performed after consultation with a radiologist/MR physicist and in accordance with MR safety guidelines [28] (please also refer to the section "MRI safety").

- Tracer ([18F]-FDG) application

- Blood glucose levels should be determined prior to [18F]FDG injection. In case of hyperglycemia, [18F]-FDG uptake into the tumor may be decreased. Hence, in case the glucose level is above $150-200 \mathrm{mg} / \mathrm{dl}$, the examination should either be rescheduled or appropriate insulin medication (including monitoring of blood glucose levels to ensure appropriate levels) should be considered [6].

- Patients should rest comfortably in a reclining chair or on a bed. Patients should not speak or engage in physical activity during the uptake time of the tracer following tracer injection.

- Please refer to the guidelines in Nuclear Medical Imaging (AWMF Guidelines Register 031/030) regarding general precautions for the application of [18F]-FDG [29].

- Prior to the imaging examination, patients should be asked to void.

\section{MRI safety}

The following points relevant to MRI safety in PET/MRI are to be considered:

- For patient safety, all patients should be routinely checked and screened with standardized checklists for potential MR contraindications (e. g. pregnancy, previous contrast agent reactions, catheters, ports, metallic implants, vascular stents, active implants, cardiac pacemakers, etc.) [30].

- All metal objects (e. g. dental prostheses, clothing with zippers and buttons) should be removed from the patient and cotton clothing without metal should be provided to the patient.

- Regarding implants, the specific kind of implant, its location, and its material need to be investigated beforehand. Information about the MR compatibility and safety of an implant can be assessed from the implant pass and/or directly from the implant/device manufacturer (e. g. online sources). The following safety regulations apply and should be adhered to: "MR unsafe" - absolute contraindication; "MR conditional” - relative contraindication, conditions apply; "MR safe" - no contraindication. In case of "MR conditional" implants, all conditions (e. g. max. field strength, SAR limitations, etc.) as provided by the implant manufacturer and online sources must be reviewed and applied during the MRI (PET/MRI) examination. In case of "MR unsafe" implants, the indication for the PET/MRI examination needs to be scrutinized and other imaging options should be considered.

- Beyond safety concerns, implants may cause artifacts, largevolume signal voids and geometric distortions in MR imaging. This may hamper image interpretation.

\section{Attenuation correction}

In contrast to CT-based attenuation correction (AC) in PET/CT [31], the attenuation properties of tissue cannot be derived directly from complementary MR images. Therefore, different concepts of MR-based attenuation correction have been introduced [24]. The most commonly applied method is based on a twopoint Dixon technique, which facilitates a 4-compartment-model attenuation map ( $\mu$-map) to identify air, lung tissue, fat, and soft tissue [32-34]. Based on this segmentation of MR images into distinct tissue classes, the individual compartments are assigned a predefined linear attenuation coefficient (LAC) for the corresponding tissue [33, 35, 36].

A number of challenges including the systematic underestimation of PET quantification related to standard MR-based attenuation correction have been reported, the most prominent being the lack of consideration of bone tissue and the occurrence of truncation artifacts [36, 37] (for further information please refer to the section "Artifacts"). Different compensation approaches for brain and whole-body imaging have been proposed to account for the misclassification of bone tissue as soft tissue [38, 39]. Promising results for whole-body imaging were shown when utilizing a CT-based 3-dimensional bone-model of major bones as an adjunct to MR-based AC data [34, 40 -42].

\section{Artifacts}

Following the introduction of integrated PET/MRI systems, a number of artifacts have been reported that are related to PET-only, MRI-only or integrated PET/MRI acquisitions. A selection of the most common artifacts and potential solutions is discussed in the following paragraph [25].

The most evident artifacts have been shown to be related to MR-based attenuation correction, causing a systematic underestimation of PET quantification when compared to PET/CT [43, 44]. Apart from the misclassification of bone tissue (please refer to the section "Attenuation correction"), truncation artifacts are a major concern in integrated PET/MRI. Due to the limited transaxial and lateral field of view (FOV) in MR imaging to a spherical diameter of about $50 \mathrm{~cm}$, structures beyond these dimensions show geometric distortions and signal voids, resulting in truncation artifacts alongside the patient arms and incorrect PET quantification $[25,45]$. In addition to the PET-based MLAA algorithm (maximum likelihood estimation of attenuation and activity) deriving the patients outer body contours from PET data $[46,47]$, a novel purely MR-based truncation correction method was introduced by Blumhagen et al. [48, 49]. This method, also referred to as HUGE (B0 homogenization using gradient enhancement), enlarges the lateral FOV in MR imaging beyond the conventional $50 \mathrm{~cm}$ diameter, effectively eliminating truncation artifacts along the patients arms in MR-based attenuation correction [48, 50].

Involuntary patient and organ motion causing a misalignment of emission and attenuation data is a known challenge in PET/CT imaging that may be further enhanced in PET/MR imaging due to prolonged examination times. Unlike in PET/CT and owing to simultaneous PET and MR data acquisition, PET/MRI has potential for MR-based motion correction of PET data. Different methods for motion correction have been proposed to account e.g. for 
respiratory motion artifacts including real-time MR imaging and 4D MR data of breathing motion or free-breathing MR imaging to retrospectively perform motion correction [51 - 53].

The following points relevant to MR-based attenuation correction and artifacts in PET/MRI are to be considered:

- In PET/MRI, AC is based on MR imaging. Thus, artifacts in MRAC have a direct effect on PET quantification. Consequently, MR-based AC needs to be accurate and free of artifacts to provide precise PET quantification. MR-AC images shall be routinely checked for artifacts, consistency and plausibility during $\mathrm{PET} / \mathrm{MR}$ image reading. Typical artifacts are mis-segmentation of air/soft tissue/fat/bone and metal artifacts due to dental prostheses and due to metallic implants such as stents and surgical clips, etc. Artifacts may be displayed as signal voids, exceeding the true dimensions of metal inclusions. Thus, artifacts are mostly easily detectable in MR-AC, indicating regions of potentially inaccurate PET quantification [45, 54].

- While new features for the improvement of MR-AC are constantly developed and implemented into the commercial software of available PET/MRI systems, including high-resolution Dixon AC, ultrashort echo time (UTE), zero TE (ZTE) sequences and/or bone models for bone detection in PET/MRI attenuation correction [17, 34, 40, 41], users need to remain attentive to MR-AC related limitations and artifacts in SUV quantification.

- Truncation artifacts along patient arms in MR-AC may affect PET quantification. The standard method on all available PET/ MRI systems for truncation correction is the PET-based MLAA algorithm [46]. A more recent method for improved MR-based truncation correction in MR-AC is HUGE $[41,48,50]$.

- Only radiofrequency (RF) coils that are labelled for combined PET/MRI use should be used. Using standard RF coils that are labelled for MR-only use will not be considered in PET/MRI AC and may, thus, lead to inaccurate PET quantification and artifacts in PET [32, 55].

\section{Quality control}

Quality control of PET tracers is governed by the "Draft Guidelines for Radiopharmacy" [56]. Quality control and application recommendations for MR contrast agents are addressed in the guidelines of the European Society of Urogenital Radiology [26]. Quality control procedures for the PET and MRI subsystems should be set up in accordance with the published guidelines $[57,58]$ but shall at least follow the vendor's recommendations. In addition, proper cross-calibration of the PET system with the respective dose calibrator has to be ensured. In routine operation, daily quality control scans (using a dedicated phantom) shall be conducted prior to patient scans to ensure correct PET acquisition and quantification.

\section{Imaging workflows}

Imaging workflows may vary with the clinical indication. Similar to $\mathrm{PET} / \mathrm{CT}$ imaging in oncology, PET/MRI can be performed in wholebody mode, meaning that patients are scanned over multiple, consecutive bed positions to cover larger co-axial imaging ranges. Given the extensive variability of MR imaging protocols and the choice of MR sequences, whole-body PET/MRI examinations have been shown to take longer than PET/CT examinations of the same co-axial imaging range. Therefore, the need for optimized and standardized PET/MR imaging workflows has become widely recognized. Over the past years, a number of proposals have been published $[59,60]$. This document sets out to describe suitable imaging conditions and protocols for whole-body [18F]-FDG$\mathrm{PET} / \mathrm{MRI}$ of oncology patients. Of note, specific protocols and MR sequences are subject to change depending on the user, vendor and indication for the examination.

For reasons of simplification and conformity to PET/CT imaging, all workflows mentioned below apply to whole-body coverage from skull-base to mid-thighs. This coverage is usually achieved within four to five bed positions (BP) depending on the patient height. Accordingly, a combination of dedicated (attenuation-corrected) radiofrequency (RF) head and neck coils and a varying number of phased-array body surface RF coils are utilized as needed [32]. Imaging is commonly performed in a supine position starting from mid-thigh to skull-base to ensure minimal impairment of lesions in the vicinity of the bladder due to increased [18F]-FDG activity in the bladder.

In a first step MRI localizers are acquired to define the axial range for the examination. Pre-scanning of the shimming and adjustment of the magnetic field are followed by the attenuation correction (AC) sequence for every BP (for detailed information regarding MR-based $A C$ please refer to section "Attenuation correction").

\section{Workflow 1: Ultra-fast PET/MRI}

This workflow is based on a 2-min/BP acquisition that facilitates ultra-fast "PET/CT-like" whole-body staging within a total time of just under $20 \mathrm{~min}$ [61]. The reasoning for this specific algorithm is to facilitate ultra-fast whole-body staging, e. g., in patients with low compliance (e. g. elderly, pediatric) or as a whole-body coverage adjunct to local dedicated imaging (e.g., local dedicated tumor staging in head and neck cancer or soft tissue sarcoma + whole-body ultra-fast).

Indications for this ultra-fast workflow include whole-body staging, e.g., for lymphoma or staging and exclusion of relapse of tumors.

Potential sequences to be obtained within the 2-min PET include: (1) Fast T2-weighted spin echo sequence (e. g. HASTE) and (2) nonenhanced fast fat-saturated T1-weighted gradient echo sequence (e. g. VIBE). Contrast media injection and acquisition of post-contrast fast $\mathrm{T} 1$-weighted fat-saturated imaging may be performed subsequent to the non-enhanced sequences. In case of primary tumors (e. g. malignant melanoma, neuroendocrine tumors) known to cause hyperarterialized metastases of the parenchymatous organs, additional dynamic contrast-enhanced imaging of the upper abdomen (e.g. VIBE) can be added. The combination of the sequences above enables the combined assessment of T2, non-enhanced and contrast-enhanced features of potential lesions ( $\vee$ Fig. 1).

\section{Workflow 2: Fast PET/MRI}

This workflow is based on a 4-min/bed acquisition that comprises diffusion-weighted imaging (DWI) in addition to the above-mentioned sequences listed in the ultra-fast algorithm ( $>$ Fig. 2) [62, 63]. The additional diffusion-weighted sequence offers comple- 


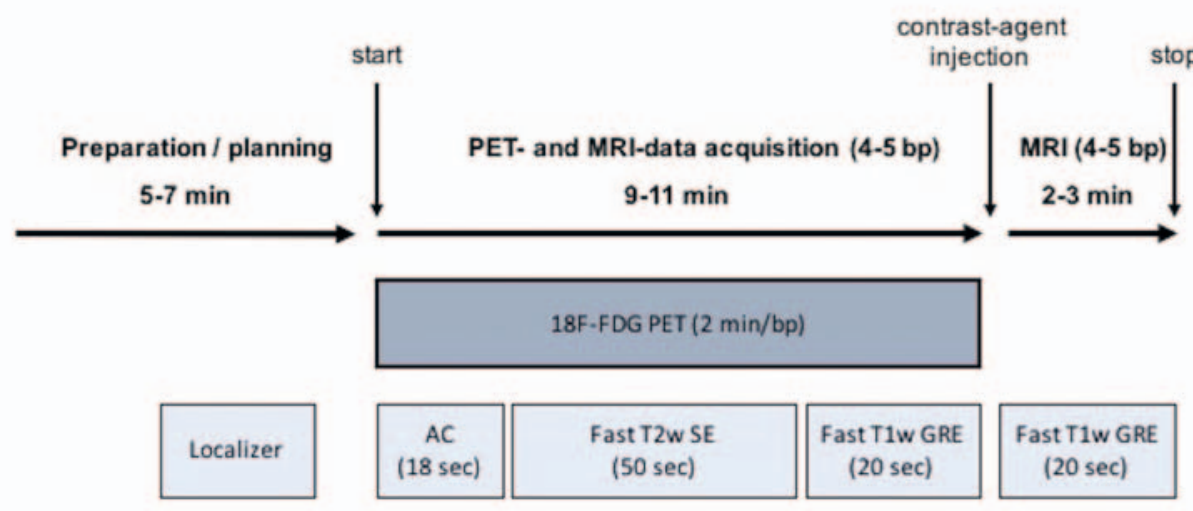

Fig. 1 Schematic workflow for the ultra-fast whole-body [18F]-FDG-PET/MRI.
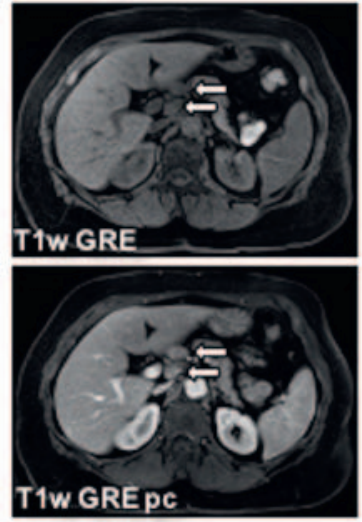

ULTRA FAST - PET/MRI
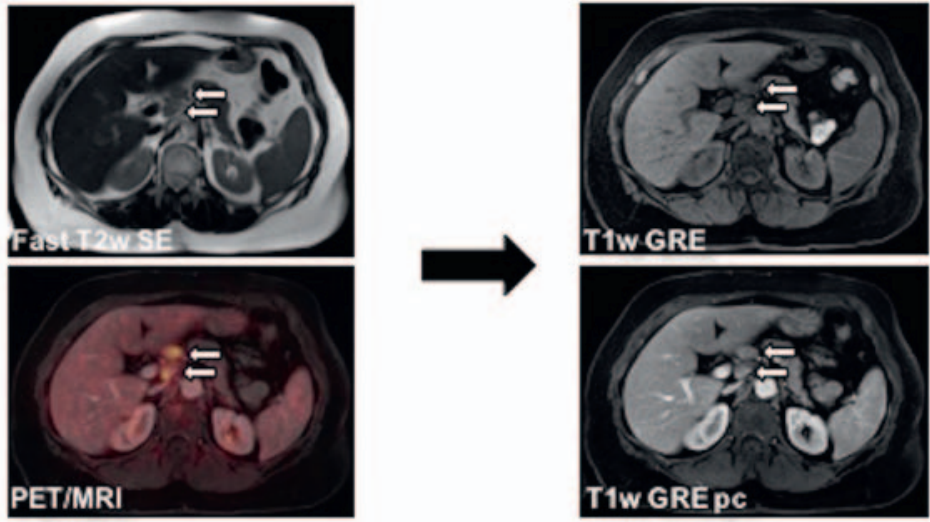

FAST - PET/MRI

- Fig. 2 Imaging example of a 45-y/o patient with a celiac lymph node metastasis (white arrows) imaged in ultra-fast and fast [18F]-FDG-PET/MRI.

mentary tissue information to PET and may be applied as a "search" sequence as it is considered useful particularly in the detection of small lesions, e. g., liver metastases that may be too small to be picked up by PET. Together with potential post-contrast T1w gradient echo sequences, this "fast PET/MRI" algorithm should require less than 30 min depending on the total amount of $\mathrm{BP}$ and duration of shimming, etc. ( $\triangleright$ Fig. 3 ).

\section{Workflow 3: Dedicated local and whole-body PET/MRI}

This workflow comprises dedicated local PET/MRI of the tumor region (e. g., head and neck, cervical cancer, soft tissue sarcoma of the limbs) and fast sequences for whole-body coverage. The aim of this workflow is to facilitate a dedicated workup of the primary cancer and whole-body staging in one examination. The MR protocol for the dedicated local PET/MRI scan should be selected in accordance with the primary tumor and guideline recommendations (e. g., cervical cancer [64]). Whole-body imaging can be performed utilizing the above-named ultra-fast or fast algorithm depending on patient compliance, potential benefit derived from DWI and desired length of the examination ( $>$ Fig. 4, 5).

\section{Reading and reporting}

The following recommendations on reading and reporting are intended to serve as assistance to novice PET/MRI readers and help standardization. High quality reading and reporting of PET/MRI examinations is based on expert knowledge of PET and MRI imaging 


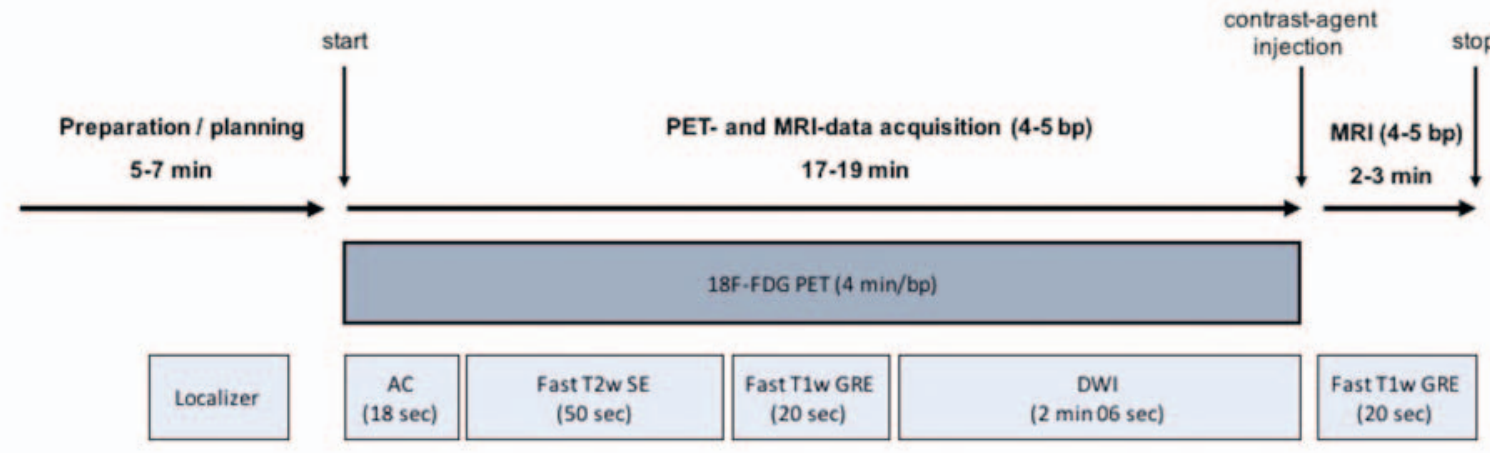

Fig. 3 Schematic workflow for fast whole-body [18F]-FDG-PET/MRI.

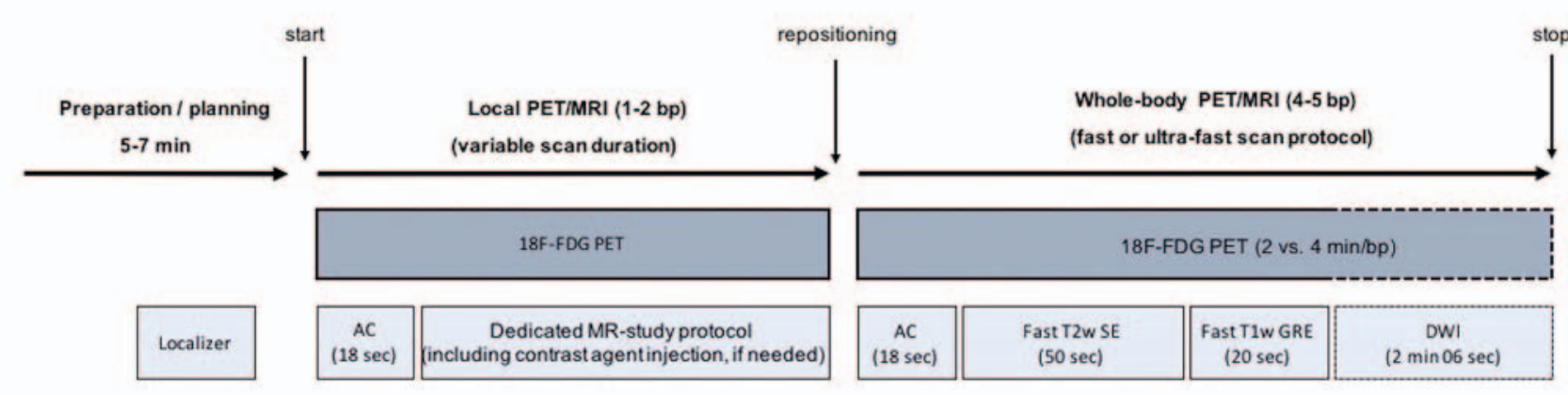

- Fig. 4 Schematic workflow of dedicated local [18F]-FDG-PET/MRI + whole-body staging.

\section{a}
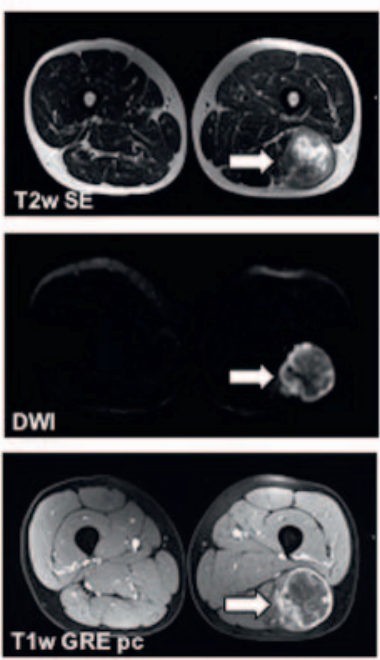
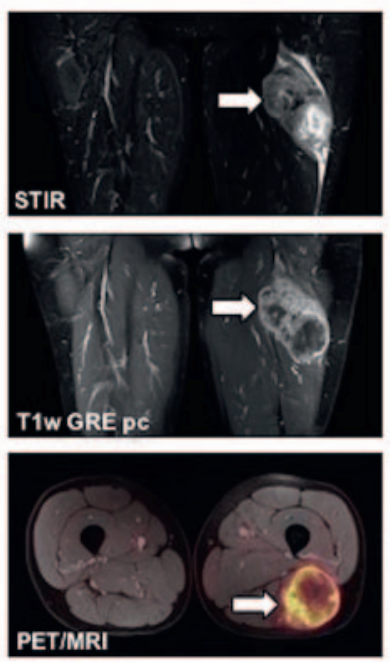

b
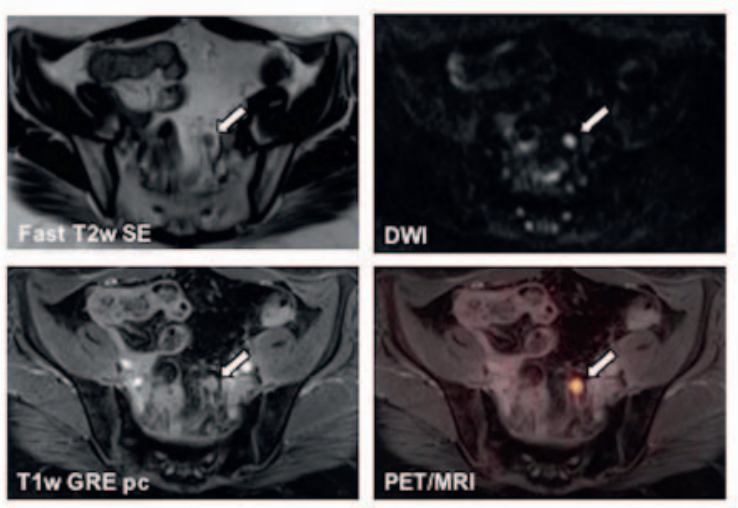

- Fig. 5 Imaging example of a [18F]-FDG-PET/MRI scan of a 52-y/o patient with a large soft tissue sarcoma of the left lower limb (thick arrows). The figure displays the dedicated local PET/MRI protocol a for assessment of the primary tumor and the fast protocol for whole-body staging $\mathbf{b}$, revealing an iliac lymph node metastasis in the left hemipelvis.

[65]. Hence, PET/MRI reading should be performed jointly by a radiologist and a nuclear medicine physician or by adequately trained dual-certified physicians (nuclear medicine and radiology).
It is important to evaluate the "raw" MRI and PET data as well as fused imaging. In contrast to rather distinct differences in the required expertise and duration of reading MRI versus $C T, P E T /$ 
MRI reporting can be conducted similar to PET/CT reporting. After reporting of the definition of the exam, clinical information, examination procedure and key parameters (including the applied radioactivity, uptake time and amount of contrast agent), the actual report, in terms of imaging findings and their evaluation, can be written as an integrated (conjoint description and evaluation of findings in MRI and PET) or separate report (subsequent description of findings in MRI and PET and conjoint evaluation).

\section{Conclusion}

Since its introduction in 2010, whole-body PET/MRI has become well-established in scientific and clinical imaging. Still, a number of basic, methodological and professional challenges have limited its wider general acceptance in the oncologic community as well as its utilization as a diagnostic alternative to PET/CT. The greatest obstacle is caused by extensive and heterogenous protocols that have rendered $\mathrm{PET} / \mathrm{MRI}$ a research tool that is incompatible with clinical use and is economically challenging. Thus, we introduced recommendations on workflow options that offer efficient and fast imaging protocols open for adaptation to meet the purpose of the examination. The three categories of imaging protocols above allow the standardization and harmonization of PET/MRI, which is a prerequisite for multi-center trials and the assessment of large patient cohorts. This may support the future adoption of $\mathrm{PET} / \mathrm{MRI}$ in clinical routine imaging and institute reimbursement.

\section{Conflict of Interest}

Dr. Herrmann reports personal fees from Bayer, other from Sofie Biosciences, personal fees from SIRTEX, other from ABX, personal fees from Adacap, personal fees from Curium, personal fees from Endocyte, grants and personal fees from BTG, personal fees from IPSEN, personal fees and non-financial support from Siemens Healthineers, non-financial support from GE Healthcare, outside the submitted work.

The other authors declare that they have no conflict of interest.

\section{References}

[1] Beyer T, Freudenberg LS, Czernin J et al. The future of hybrid imaging-part 3: PET/MR, small-animal imaging and beyond. Insights Imaging 2011; 2: 235-246. doi:10.1007/s13244-011-0085-4

[2] Gambhir SS, Czernin J, Schwimmer ] et al. A tabulated summary of the FDG PET literature. J Nucl Med 2001; 42: 1S-93S

[3] Smith TA. FDG uptake, tumour characteristics and response to therapy: a review. Nucl Med Commun 1998; 19: 97-105

[4] Kapoor V, McCook BM, Torok FS. An introduction to PET-CT imaging. Radiographics 2004; 24: 523-543. doi:10.1148/rg.242025724

[5] Petersen $\mathrm{H}$, Holdgaard PC, Madsen PH et al. FDG PET/CT in cancer: comparison of actual use with literature-based recommendations. Eur J Nucl Med Mol Imaging 2016; 43: 695 - 706. doi:10.1007/s00259-015-3217-0

[6] Boellaard R, Delgado-Bolton R, Oyen W] et al. FDG PET/CT: EANM procedure guidelines for tumour imaging: version 2.0. Eur J Nucl Med Mol Imaging 2015; 42: 328 -354. doi:10.1007/s00259-014-2961-x

[7] Pooley RA. AAPM/RSNA physics tutorial for residents: fundamental physics of MR imaging. Radiographics 2005; 25: 1087 - 1099. doi:10.1148/ rg. 254055027
[8] Kuhl CK, Gieseke J, von Falkenhausen M et al. Sensitivity encoding for diffusion-weighted MR imaging at 3.0 T: intraindividual comparative study. Radiology 2005; 234: 517 - 526. doi:10.1148/radiol.2342031626

[9] Nensa F, Beiderwellen K, Heusch P et al. Clinical applications of PET/MRI: current status and future perspectives. Diagn Interv Radiol 2014; 20 : 438 - 447. doi:10.5152/dir.2014.14008

[10] Pichler BJ, Kolb A, Nagele T et al. PET/MRI: paving the way for the next generation of clinical multimodality imaging applications. J Nucl Med 2010; 51: 333 - 336. doi:10.2967/jnumed.109.061853

[11] Wehrl HF, Sauter AW, Divine MR et al. Combined PET/MR: a technology becomes mature. J Nucl Med 2015; 56: 165 - 168. doi:10.2967| jnumed.114.150318

[12] Wehrl HF, Wiehr S, Divine MR et al. Preclinical and Translational PET/MR Imaging. J Nucl Med 2014; 55: 11S-18S. doi:10.2967| jnumed.113.129221

[13] Ziegler SI, Pichler BJ, Boening G et al. A prototype high-resolution animal positron tomograph with avalanche photodiode arrays and LSO crystals. Eur J Nucl Med 2001; 28: 136-143

[14] Schlemmer HP, Pichler BJ, Schmand M et al. Simultaneous MR/PET imaging of the human brain: feasibility study. Radiology 2008; 248: 1028 1035. doi:10.1148/radiol.2483071927

[15] Spick C, Herrmann K, Czernin J. 18F-FDG PET/CT and PET/MRI Perform Equally Well in Cancer: Evidence from Studies on More Than $2300 \mathrm{~Pa}$ tients. J Nucl Med 2016; 57: 420 -430. doi:10.2967/jnumed.115.158808

[16] Czernin J, Ta L, Herrmann K. Does PET/MR Imaging Improve Cancer Assessments? Literature Evidence from More Than 900 Patients. J Nucl Med 2014; 55: 59S-62S. doi:10.2967/jnumed.114.141838

[17] Bailey DL, Pichler B], Guckel B et al. Combined PET/MRI: Global Warming-Summary Report of the 6th International Workshop on PET/MRI, March 27-29, 2017, Tubingen, Germany. Mol Imaging Biol 2018; 20: 4 20. doi:10.1007/s11307-017-1123-5

[18] Sawicki LM, Grueneisen J, Schaarschmidt BM et al. Evaluation of (1)(8)FFDG PET/MRI, (1)(8)F-FDG PET/CT, MRI, and CT in whole-body staging of recurrent breast cancer. Eur J Radiol 2016; 85: 459-465. doi:10.1016/j.ejrad.2015.12.010

[19] Schaarschmidt BM, Grueneisen J, Heusch P et al. Does 18F-FDG PET/MR reduce the number of indeterminate abdominal incidentalomas compared with 18F-FDG PET/CT? Nucl Med Commun 2015; 36: 588 - 595. doi:10.1097/MNM.0000000000000298

[20] Queiroz MA, Kubik-Huch RA, Hauser $N$ et al. PET/MRI and PET/CT in advanced gynaecological tumours: initial experience and comparison. Eur Radiol 2015; 25: 2222 - 2230. doi:10.1007/s00330-015-3657-8

[21] Fendler WP, Czernin J, Herrmann K et al. Variations in PET/MRI Operations: Results from an International Survey Among 39 Active Sites. J Nucl Med 2016; 57: 2016 - 2021. doi:10.2967/jnumed.116.174169

[22] Krause B], Beyer T, Bockisch A et al. FDG-PET/CT in oncology. German Guideline. Nuklearmedizin 2007; 46: 291 - 301

[23] Delbeke D, Coleman RE, Guiberteau MJ et al. Procedure guideline for tumor imaging with 18F-FDG PET/CT 1.0. J Nucl Med 2006; 47: 885-895

[24] Wagenknecht G, Kaiser H], Mottaghy FM et al. MRI for attenuation correction in PET: methods and challenges. MAGMA 2013; 26: 99-113. doi:10.1007/s10334-012-0353-4

[25] Keller SH, Holm S, Hansen AE et al. Image artifacts from MR-based attenuation correction in clinical, whole-body PET/MRI. MAGMA 2013; 26: 173 - 181. doi:10.1007/s10334-012-0345-4

[26] European Society of Urogenital Radiology (ESUR). ESUR Guidelines: 10.0 Contrast Media Safety Guidelines. 2018. Avalable from: http://www. esur.org/esur-guidelines/

[27] European Medicines Agency (EMA). Gadolinium containing contrast agents - Assessment report - EMEA/H/A-31/1437. 2017. Available from: https://www.ema.europa.eu/documents/referral/gadolinium-arti cle-31-referral-assessment-report_en.pdf 
[28] Sammet S. Magnetic resonance safety. Abdom Radiol (NY) 2016; 41: 444 - 451. doi:10.1007/s00261-016-0680-4

[29] Leitlinienreport zur DGN-Handlungsempfehlung (S1-Leitlinie). Nuklearmedizinische Bildgebung. Stand: 01/2015 - AWMF-Registernummer: 031-030. Available from: https://www.awmf.org/leitlinien/detail/I/031-030.html

[30] Shellock FG. Reference Manual for Magnetic Resonance Safety, Implants and Devices 2018. Shellock R \& D Services. 2018

[31] Kinahan PE, Townsend DW, Beyer T et al. Attenuation correction for a combined 3D PET/CT scanner. Med Phys 1998; 25: 2046-2053. doi:10.1118/1.598392

[32] Quick HH. Integrated PET/MR. J Magn Reson Imaging 2014; 39: 243 258. doi:10.1002/jmri.24523

[33] Martinez-Moller A, Souvatzoglou M, Delso G et al. Tissue classification as a potential approach for attenuation correction in whole-body PET/MRI: evaluation with PET/CT data. J Nucl Med 2009; 50: 520 - 526. doi:10.2967/jnumed.108.054726

[34] Paulus DH, Quick HH, Geppert C et al. Whole-Body PET/MR Imaging: Quantitative Evaluation of a Novel Model-Based MR Attenuation Correction Method Including Bone. J Nucl Med 2015; 56: 1061 - 1066. doi:10.2967/jnumed.115.156000

[35] Schulz V, Torres-Espallardo I, Renisch S et al. Automatic, three-segment, MR-based attenuation correction for whole-body PET/MR data. Eur J Nucl Med Mol Imaging 2011; 38: 138-152. doi:10.1007/s00259-010-1603-1

[36] Beyer T, Lassen ML, Boellaard R et al. Investigating the state-of-the-art in whole-body MR-based attenuation correction: an intra-individual, intersystem, inventory study on three clinical PET/MR systems. MAGMA 2016; 29: 75-87. doi:10.1007/s10334-015-0505-4

[37] Samarin A, Burger C, Wollenweber SD et al. PET/MR imaging of bone lesions-implications for PET quantification from imperfect attenuation correction. Eur J Nucl Med Mol Imaging 2012; 39: 1154-1160. doi:10.1007/s00259-012-2113-0

[38] Yang J, Jian Y, Jenkins N et al. Quantitative Evaluation of Atlas-based Attenuation Correction for Brain PET in an Integrated Time-of-Flight PET/MR Imaging System. Radiology 2017; 284: 169-179. doi:10.1148/ radiol.2017161603

[39] Yang J, Wiesinger F, Kaushik S et al. Evaluation of Sinus/Edge-Corrected Zero-Echo-Time-Based Attenuation Correction in Brain PET/MRI. J Nucl Med 2017; 58: 1873 - 1879. doi:10.2967/jnumed.116.188268

[40] Koesters T, Friedman KP, Fenchel $M$ et al. Dixon Sequence with Superimposed Model-Based Bone Compartment Provides Highly Accurate PET/MR Attenuation Correction of the Brain. J Nucl Med 2016; 57: 918 924. doi:10.2967/jnumed.115.166967

[41] Oehmigen M, Lindemann ME, Gratz M et al. Impact of improved attenuation correction featuring a bone atlas and truncation correction on PET quantification in whole-body PET/MR. Eur ] Nucl Med Mol Imaging 2018; 45: 642 - 653. doi:10.1007/s00259-017-3864-4

[42] Elschot M, Selnaes KM, Johansen $\mathrm{H}$ et al. The Effect of Including Bone in DIXON-based Attenuation Correction for (18)F-fluciclovine PET/MRI of Prostate Cancer. J Nucl Med 2018. doi:10.2967/jnumed.118.208868

[43] Drzezga A, Souvatzoglou M, Eiber M et al. First clinical experience with integrated whole-body PET/MR: comparison to PET/CT in patients with oncologic diagnoses. J Nucl Med 2012; 53: 845 - 855. doi:10.2967| jnumed.111.098608

[44] Boellaard R, Quick HH. Current image acquisition options in PET/MR. Semin Nucl Med 2015; 45: 192 - 200. doi:10.1053/j.semnuclmed.2014.12.001

[45] Brendle C, Schmidt H, Oergel A et al. Segmentation-based attenuation correction in positron emission tomography/magnetic resonance: erroneous tissue identification and its impact on positron emission tomography interpretation. Invest Radiol 2015; 50: 339-346. doi:10.1097| RLI.0000000000000131

[46] Nuyts J, Bal G, Kehren F et al. Completion of a truncated attenuation image from the attenuated PET emission data. IEEE Trans Med Imaging 2013; 32: 237 - 246. doi:10.1109/TMI.2012.2220376
[47] Nuyts ], Dupont P, Stroobants S et al. Simultaneous maximum a posteriori reconstruction of attenuation and activity distributions from emission sinograms. IEEE Trans Med Imaging 1999; 18: 393 - 403. doi:10.1109/42.774167

[48] Blumhagen JO, Braun H, Ladebeck R et al. Field of view extension and truncation correction for MR-based human attenuation correction in simultaneous MR/PET imaging. Med Phys 2014; 41: 022303. doi:10.1118/ 1.4861097

[49] Blumhagen JO, Ladebeck R, Fenchel M et al. MR-based field-of-view extension in MR/PET: B0 homogenization using gradient enhancement (HUGE). Magn Reson Med 2013; 70: 1047-1057. doi:10.1002/ mrm.24555

[50] Lindemann ME, Oehmigen M, Blumhagen JO et al. MR-based truncation and attenuation correction in integrated PET/MR hybrid imaging using HUGE with continuous table motion. Med Phys 2017; 44: 4559-4572. doi:10.1002/mp.12449

[51] Catana C. Motion correction options in PET/MRI. Semin Nucl Med 2015 45: 212 - 223. doi:10.1053/j.semnuclmed.2015.01.001

[52] Catalano OA, Umutlu L, Fuin $\mathrm{N}$ et al. Comparison of the clinical performance of upper abdominal PET/DCE-MRI with and without concurrent respiratory motion correction (MoCo). Eur J Nucl Med Mol Imaging 2018. doi:10.1007/s00259-018-4084-2

[53] Gratz M, Ruhlmann V, Umutlu L et al. Impact of MR-based motion correction on clinical PET/MR data of patients with thoracic pathologies. In proc. ISMRM 2017, Apr 21-27 Honolulu, HI, USA, 2017: 3899

[54] Fuin N, Pedemonte S, Catalano OA et al. PET/MRI in the Presence of Metal Implants: Completion of the Attenuation Map from PET Emission Data. J Nucl Med 2017; 58: 840 -845. doi:10.2967/jnumed.116.183343

[55] Paulus DH, Quick HH. Hybrid Positron Emission Tomography/Magnetic Resonance Imaging: Challenges, Methods, and State of the Art of Hardware Component Attenuation Correction. Invest Radiol 2016; 51: 624 634. doi:10.1097/RLI.0000000000000289

[56] Eanm. Draft guidelines for radiopharmacy. Eur ] Nucl Med Mol Imaging 2003; 30: BP63 - BP72

[57] Committee EP, Busemann Sokole E, Plachcinska A et al. Routine quality control recommendations for nuclear medicine instrumentation. Eur J Nucl Med Mol Imaging 2010; 37: 662 - 671. doi:10.1007/s00259-009-1347-y

[58] International Atomic Energy Agency (IAEA). Quality Assurance for PET and PET/CT Systems. 2009. Available from https://www-pub.iaea.org/ books/iaeabooks/8002/Quality-Assurance-for-PET-and-PET-CT-Systems

[59] Martinez-Moller A, Eiber M, Nekolla SG et al. Workflow and scan protoco considerations for integrated whole-body PET/MRI in oncology. J Nucl Med 2012; 53: 1415-1426. doi:10.2967/jnumed.112.109348

[60] von Schulthess GK, Veit-Haibach P. Workflow Considerations in PET/MR Imaging. J Nucl Med 2014; 55: 19S-24S. doi:10.2967| jnumed.113.129239

[61] Kirchner ], Sawicki LM, Suntharalingam S et al. Whole-body staging of female patients with recurrent pelvic malignancies: Ultra-fast 18F-FDG PET/MRI compared to 18F-FDG PET/CT and CT. PLoS One 2017; 12 e0172553. doi:10.1371/journal.pone.0172553

[62] Grueneisen J, Schaarschmidt BM, Heubner M et al. Implementation of FAST-PET/MRI for whole-body staging of female patients with recurrent pelvic malignancies: A comparison to PET/CT. Eur J Radiol 2015; 84: 2097 - 2102. doi:10.1016/j.ejrad.2015.08.010

[63] Grueneisen J, Sawicki LM, Schaarschmidt BM et al. Evaluation of a Fast Protocol for Staging Lymphoma Patients with Integrated PET/MRI. PLoS One 2016; 11: e0157880. doi:10.1371/journal.pone.0157880

[64] Marth C, Landoni F, Mahner S et al. Cervical cancer: ESMO Clinical Practice Guidelines for diagnosis, treatment and follow-up. Ann Oncol 2017; 28: iv72 - iv83. doi:10.1093/annonc/mdx220

[65] Stegger L, Schafers M, Weckesser M et al. EANM-ESR white paper on multimodality imaging. Eur J Nucl Med Mol Imaging 2008; 35: 677 680. doi:10.1007/s00259-008-0724-2 